\title{
Interval between preexposure and test determines the magnitude of latent inhibition: Implications for an interference account
}

\author{
LUIS AGUADO \\ Universidad Complutense de Madrid, Madrid, Spain \\ and \\ MICHELLE SYMONDS and GEOFFREY HALL \\ University of York, York, England
}

\begin{abstract}
The effect of a retention interval on latent inhibition was studied in three experiments by using rats and the conditioned taste-aversion procedure. In Experiment 1, we demonstrated an apparent loss of latent inhibition (i.e., a strengthening of the aversion) in preexposed subjects that experienced a retention interval of 12 days between conditioning and the test. In Experiment 2, we found no effect of this retention interval on the habituation of neophobia produced by the phase of exposure to the flavor. In Experiment 3, we showed that interposing a retention interval between preexposure and conditioning produced effects exactly comparable to those seen in Experiment 1. The implications of these results for rival theories of latent inhibition, as an acquisition deficit or as a case of interference at retrieval, are discussed.
\end{abstract}

Latent inhibition is the retardation of conditioning observed when the to-be-conditioned stimulus (CS) is preexposed before the conditioning phase (Lubow, 1973). This effect has usually been thought of as reflecting an acquisition deficit, variously attributed to a decrease in the associability of the stimulus (Mackintosh, 1975; Pearce \& Hall, 1980), the conditioning of inattention (Lubow, Weiner, \& Schnur, 1981), or reduced processing of the stimulus caused by priming by the context in which it has been exposed (Wagner, 1976). For all these theories, the reduced CR (conditioned response) observed after conditioning with a preexposed stimulus is assumed to reflect a weak underlying association. It is possible, however, that latent inhibition might be the result, in whole or in part, not of an acquisition deficit, but of a failure of retrieval. For example, Bouton (1991) and Kraemer and Roberts (1984) have suggested that the preexposure and conditioning experiences generate independent memories, and that although the association of the preexposed stimulus with the US (unconditioned stimulus) is perfectly well established, the memory of the preexposure experience interferes with its retrieval at the time of testing.

Support for the interference account has been sought in attempts to show that certain procedures can act as

\footnotetext{
This research was supported by a grant from the Spanish Ministerio de Educacion y Ciencia (Estancias de Investigadores en Centros de Investigacion Extranjeros) to the first author, and by a grant from the U.K. Science and Engineering Research Council to the third author. We thank Charlotte Bonardi for her helpful comments. Address correspondence to G. Hall, Department of Psychology, University of York, YO1 5DD England.
}

"reminders" that will promote the retrieval of one memory over another. Thus, Kasprow, Catterson, Schachtman, and Miller (1984) found that reexposing an animal to the US before the test phase of a latent inhibition experiment resulted in a more vigorous $\mathrm{CR}$, a result they interpreted as suggesting that the reminder treatment could attenuate the interfering effects of the preexposure phase and ensure expression of the CS-US association established during conditioning. A converse effect, reinstatement of latent inhibition, has been recently reported by Ackil, Carman, Bakner, and Riccio (1992). These authors found that although preexposure to sucrose 10 days before taste-aversion conditioning did not reduce the strength of the acquired aversion, a "reminder" presentation of sucrose the day before conditioning restored the effects of preexposure; that is, latent inhibition was found under these conditions. Such a reinstatement of latent inhibition might be expected if this reminder treatment were able to reactivate the memory of the preexposure phase of the procedure.

A second line of evidence, and one that forms the focus of the present experiments, comes from studies of the effects of imposing a long retention interval between conditioning and testing in a latent inhibition procedure. Using the conditioned taste-aversion procedure, Kraemer and Roberts (1984) found that, in some conditions of training, the aversion to the CS in the preexposed animals was stronger when the test was given 21 days after conditioning rather than on the day following conditioning. This result is not to be expected on the basis of the notion that latent inhibition is an acquisition deficit-a weak association present immediately after conditioning would not 
be expected to grow stronger with the passage of time. Kraemer and Roberts propose, rather, that the CS-US association is well formed, even after latent inhibition training, and is not influenced substantially by the retention interval. But the interfering memory produced by preexposure becomes, it is suggested, less effective over the course of the retention interval, with the result that the latent inhibition effect is no longer evident after 21 days.

Given its potential theoretical importance, we thought it worthwhile, as our first experiment, to attempt to confirm the reliability of the effect reported by Kraemer and Roberts (1984), and also, by introducing a number of procedural modifications, to extend its generality. In Experiments 2 and 3, we initiate an analysis of the mechanisms responsible for the effect seen in Experiment 1. Experiment 2 was designed to rule out the possibility that the retention interval might have its effect by virtue of its influence on the rat's neophobic response to flavor stimuli. In Experiment 3, we tested an implication of the interference interpretation of the retention interval effect-that the critical interval in producing an attenuation of latent inhibition is that between preexposure and test, and that the interval between the conditioning trial and the test is immaterial.

\section{EXPERIMENT 1}

In previous studies of the attenuation of latent inhibition in flavor-aversion conditioning, an effect of the conditioning-to-test interval has been demonstrated only under a restricted set of conditions. In particular, a reliable effect has been observed only when the flavor used as the CS has been somewhat different from that presented during preexposure (Kraemer \& Ossenkopp, 1986; Kraemer \& Roberts, 1984; Kraemer \& Spear, 1992). Demonstrations of the effect with the same flavor in both phases are less secure. Bakner, Strohen, Nordeen, and Riccio (1991) were successful using sucrose as the flavor, as were Kraemer and Roberts and Kraemer, Hoffmann, and Spear (1988) using chocolate milk. Kraemer and Ossenkopp, however, found perfect retention of the latent inhibition effect when chocolate milk was used for both phases-an attenuation was found only when preexposure was to saccharin and conditioning was with chocolate milk. We do not know why the retention interval effect should show such sensitivity to the nature of the flavors used, and we were keen to determine if we could obtain the effect with our standard training procedures with the flavor (saccharin) that we have routinely used in our previous work on latent inhibition, and by using the same flavor both in preexposure and as the CS.

Following Kraemer and Roberts (1984), the retention intervals (the intervals between conditioning and the test) most often used in studies of the attenuation of latent inhibition have been 1 day and 21 days. In the present experiment, we reduced the longer interval to 12 days because the results of Bakner et al. (1991) indicate little effect of extending the interval beyond this point. We also increased the shorter interval to 2 days. We have found, in pilot work, that an injection of lithium chloride ( $\mathrm{LiCl})$, the US employed in the present experiments, produces transient changes in the rat's readiness to consume fluids. There is a pronounced suppression of water consumption for several hours following injection, which is sometimes followed by a compensatory increase in consumption on the following day. Variations of this sort might make it difficult to obtain an accurate measure of the strength of a conditioned aversion when the test is given within $24 \mathrm{~h}$ of conditioning. But our pilot work has shown that consumption of water returns to normal levels when an interval of $48 \mathrm{~h}$ is allowed to elapse following an $\mathrm{LiCl}$ injection.

A further, minor procedural modification was that in our experiment, the subjects were given a fixed and limited amount of fluid on each trial of preexposure and conditioning. All the animals consumed all the fluid on each presentation, so exposure to the flavor was equivalent in all training conditions. We were thus able to avoid the problems noted by Bakner et al. (1991) in their Experiment 1 (but not in their Experiment 2), in which there were substantial differences among the groups in the amounts of fluid consumed during training.

The experimental design included four groups. Two groups (Pre-2 and Pre-12) received preexposure to saccharin before a conditioning trial in which consumption of this flavor was followed by $\mathrm{LiCl}$-induced illness. The strength of the aversion generated by this treatment was tested either 2 or 12 days after the conditioning day. We hoped to show a stronger aversion in Group Pre-12 than in Group Pre-2. It is of theoretical importance to show that any such effect of retention interval is restricted to subjects that received the initial latent inhibition training. (And there is evidence that, in some circumstances, the measured strength of an aversion can increase over a retention interval, even in subjects that have had no preexposure; e.g., Batsell \& Best, 1992.) Accordingly, we included control groups, Cont-2 and Cont-12, that did not receive preexposure, but experienced the short and long retention intervals between conditioning and the test. These two groups should not differ in their test performance if the retention interval has its effects solely by acting on the memory estabished by stimulus preexposure.

\section{Method}

Subjects and Apparatus. The subjects were 32 male Lister hooded rats, with a mean weight of $500 \mathrm{~g}$ at the start of the experiment. The animals were housed individually with continuous access to food in a colony room that also served as the test room. Fluids were administered at room temperature in a $50-\mathrm{ml}$ plastic centrifuge tube with a rubber stopper fitted with a stainless steel drinking spout. In this and the following experiments, the test flavor was a solution of $0.1 \%$ sodium saccharin, and illness was induced by a $10 \%$ body-weight intraperitoneal injection of $0.3 \mathrm{M} \mathrm{LiCl}$.

Procedure. Before the preexposure phase, the animals were subjected for 4 days to a water-deprivation schedule consisting of two daily 30 -min periods of free access to water, separated by a 7-h interval. These drinking periods took place at 1100 and $1800 \mathrm{~h}$. In subsequent phases of the experiment, flavored solutions were presented during the first of these periods; the animals continued to receive water during the second period throughout the experiment. 
During the moming drinking period of each of the three preexposure days, the animals in Groups Pre-2 and Pre-12 were offered $10 \mathrm{ml}$ of the saccharin solution for $30 \mathrm{~min}$. The subjects in the control groups received $10 \mathrm{ml}$ of water in these sessions. On the next conditioning day, all received access to $10 \mathrm{ml}$ of saccharin for $30 \mathrm{~min}$, followed immediately by an injection of $\mathrm{LiCl}$. A recovery day followed, in which the animals had access to water for the usual two 30-min periods. The test consisted of three daily sessions in which the animals were allowed free access to the saccharin solution for $30 \mathrm{~min}$. For Groups Pre-2 and Cont-2, the first test was given on the day after the recovery day. For Groups Pre-12 and Cont-12, an interval of 12 days intervened between conditioning and the first test session. Training for these groups was begun 10 days before the start of training for the groups given the 2-day retention interval so as to allow the test trials for all the subjects to fall on the same days. During the last 5 days of the retention interval for the 12-day groups (the days corresponding to the preexposure, conditioning, and recovery sessions for Groups Pre-2 and Cont-2), the schedule of water administration was matched to that determining the presentation of fluids to 2-day groups. Thus, all the groups were equated in level of deprivation at the time of testing.

\section{Results and Discussion}

Figure 1 presents the mean amounts of test solution consumed by each group on each of the 3 test days. All showed an initial aversion that declined to some extent over the course of the extinction test, but more important is the clear difference between the two preexposed groups; Group Pre-12 showed less consumption than Group Pre-2. The nonpreexposed control groups showed lower levels of consumption, consistent with the suggestion that latent inhibition occurred in the preexposed groups. There was no sign that imposing a retention interval increased the size of the measured aversion in the animals that were not given latent inhibition training. There was little difference between the two control groups, but the aversion was, if anything, slightly mored marked in Group Cont-2 than in Group Cont-12.

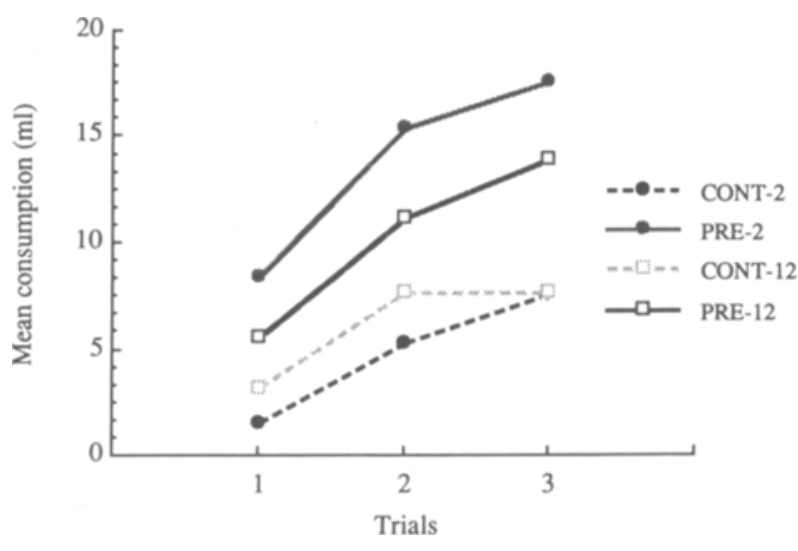

Figure 1. Mean saccharin consumption over the three test trials in Experiment 1. "Pre" groups received preexposure to the saccharin before the conditioning trial; "Cont" groups did not. For one pair of groups, the interval between conditioning and the first test trial was 2 days; for the other pair of groups, the interval was 12 days.
An analysis of variance (ANOVA) was performed on the consumption data from the test days, with interval, preexposure condition, and trials as the factors. This analysis yielded significant interactions between interval and preexposure condition $[F(1,28)=4.11, p=.05]$ and between preexposure condition and trials $[F(2,56)=6.49$, $p<.05]$. The three-way interaction of interval $\times$ preexposure $\times$ trials was not significant $(F=1.15)$. Pairwise comparisons among the group means using Duncan's test were made in order to determine the source of the interaction between interval and preexposure condition. These revealed significant differences between Groups Pre2 and Pre-12 $(p<.05)$, Pre-2 and Cont $-2(p<.01)$, and Pre-12 and Cont-12 $(p<.05)$.

This experiment confirmed that, although the latent inhibition effect is not abolished completely (at least with the 12-day interval used here), the magnitude of a conditioned taste aversion will increase over a retention interval in animals given prior latent inhibition training. Thus, these results extend the generality of the effect that was initially reported by Kraemer and Roberts (1984), and make clear that it can be found not only when different flavors are used for preexposure and conditioning (e.g., Kraemer \& Ossenkopp, 1986), but also when the same flavor is used in both phases. We cannot account for the failure of Kraemer and Roberts (1984, Experiment 3) to find the effect in groups that, like our preexposed subjects, were given three preexposure sessions with $0.1 \%$ saccharin followed by conditioning with this same flavor. The discrepancy presumably arises from some procedural difference between their experiment and ours, such as their use of scopolamine rather than $\mathrm{LiCl}$ as the US, or their use of a choice as opposed to a single-bottle test.

There was no effect of the retention interval on the aversion shown by the nonpreexposed groups; certainly there was no sign of any enhancement of the aversion in these groups (cf. Batsell \& Best, 1992), and the slight loss of the aversion in Group Cont-12 was not statistically reliable. (This last result accords with previous findings that conditioned taste aversions tend to be especially resistant to forgetting; e.g., Brookshire \& Brackbill, 1976; Colby \& Smith, 1977.) Taken as a whole, this pattern of results is consistent not only with the interference theory's claim that the preexposure experience is at least partially forgotten or becomes less retrievable over a retention interval, but also with a complementary assumption of this theory. Both Kraemer and Roberts (1984) and Bouton (1991) have suggested that memories of nonreinforced exposure to a stimulus and of the association between a stimulus and a motivationally relevant consequence are differentially sensitive to the passage of time. Specifically, it is assumed that memories of significant associations are more resistant than the memory of mere stimulus exposure. In Experiment 1 , the resistance of significant memories to forgetting is confirmed by the virtual absence of a reduction of the acquired aversion in the nonpreexposed group after a retention interval of 12 days. 


\section{EXPERIMENT 2}

The results of Experiment 1 confirmed that a retention interval will increase the magnitude of a conditioned taste aversion in subjects given initial latent inhibition training. However, before accepting an interpretation of this effect as being due to forgetting or a reduction in the retrievability of the preexposure experience, an alternative account should be considered.

One of the effects of repeated exposure to a new flavor is the habituation of the neophobic reaction initially elicited by it, a process that is reflected in a progressive increase in consumption of the flavor. Although our procedures did not allow us to observe any such effect (the subjects were allowed to consume a fixed $10 \mathrm{ml}$ of fluid on each trial), it seems very likely that the treatment given to our preexposed subjects would have produced a loss of neophobia. A rest interval without stimulation after habituation training has often been found to result in spontaneous recovery of a habituated response (e.g., Thompson \& Spencer, 1966) and there is some evidence to suggest that such recovery can occur in the case of flavor neophobia (e.g., Domjan, 1977). It is possible, then, that for our preexposed groups tested after a long retention interval, neophobia initially lost during preexposure might have returned over that interval. Then, decreased consumption on the test would not be the result of an attenuation of latent inhibition, but of recovery of neophobia.

Kraemer and Roberts (1984), in their Experiment 3, attempted to investigate the possible role of neophobia by comparing consumption of the test solution after 1 or 21 days in animals that had been exposed to the solution but that had not received an injection. However, as Lubow $(1989$, p. 185) has noted, the absence of a difference between these conditions is not enough to allow us to assert that the recovery of neophobia plays no role in the effect seen in animals given latent inhibition training. It might well be that neophobia induced by the $\mathrm{LiCl}$ injection is what recovers during the retention interval; to exclude this factor requires a control condition in which animals are not only exposed to the flavor, but also receive an unpaired injection.

This possibility has been directly evaluated in a study of the attenuation of latent inhibition after delayed testing in the conditioned emotional response procedure. Kraemer, Randall, and Carbary (1991, Experiment 2) found no effect of a 7-day retention interval on the suppression shown by subjects that had first received preexposure and then nonpaired presentations of light and an electric shock. They concluded that a recovery of unconditioned suppression was not the cause of the apparent attenuation of latent inhibition they had found in their first experiment. In the present Experiment 3, we applied a similar logic to the conditioned taste-aversion paradigm. Two groups were first preexposed to the saccharin solution and then received an $\mathrm{LiCl}$ injection unpaired with the solution. Consumption of saccharin was tested in one group after 2 days; in the other, testing was delayed for 12 days. If neophobia recovers over the retention interval, then the 12-day group should consume less on test of the solution. Such an outcome would seriously undermine the significance of the results of our previous experiment as a demonstration of the attenuation of latent inhibition with time.

\section{Method}

Subjects. The subjects were 16 male Lister hooded rats, with a mean weight of $500 \mathrm{~g}$. They were maintained on the waterdeprivation schedule that was used in Experiment 1.

Procedure. On the first 3 experimental days, all the animals were offered the saccharin solution under conditions identical to those employed for the preexposed subjects in Experiment 1. The procedure employed on the following day was similar to that used for conditioning in Experiment 1, except that the $\mathrm{LiCl}$ injection occurred in the afternoon rather than in the morning. That is, all the animals received $10 \mathrm{ml}$ of the saccharin solution in the morning and, $7 \mathrm{~h}$ later, had access to $10 \mathrm{ml}$ of water for $30 \mathrm{~min}$ followed by the $\mathrm{LiCl}$ injection. The next day was a recovery day. Test presentations of saccharin began for half the animals (Group 2) on the day following this recovery day; for the remainder (Group 12), a further 11 days intervened between the recovery day and the start of the test. As in the previous experiment, training for the subjects given the longer retention interval started earlier than training for those given the shorter interval, so that testing might occur on the same days for both groups. Procedural details not specified here were the same as those described for Experiment 1.

\section{Results and Discussion}

Figure 2 shows the mean amount of saccharin consumed by each group on the three test trials. The only sign of a difference between the two groups was on Trial 1, in which Group 2 drank slightly less than Group 12. Note, however, that this small difference was in the direction opposite from what would have been expected had neophobia recovered over the 12-day retention interval. An ANOVA was performed on the results summarized in the figure, with retention interval and trials

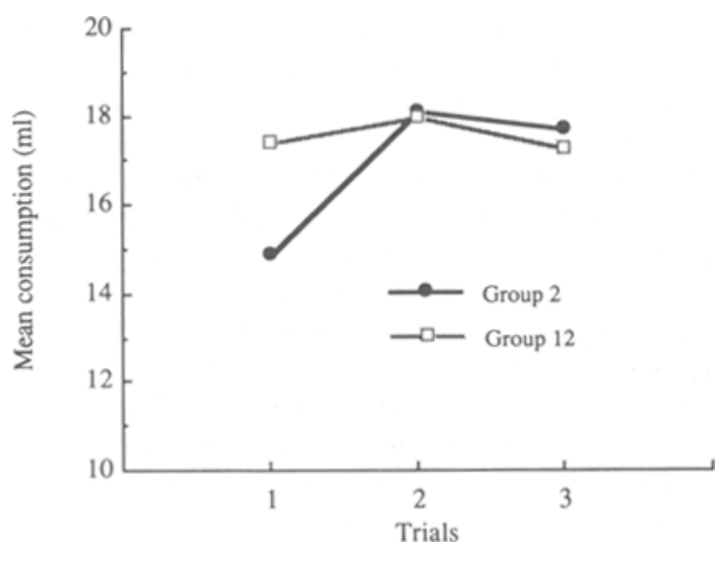

Figure 2. Mean saccharin consumption over the three test trials in Experiment 2. The subjects had received preexposure to saccharin and an unpaired injection of $\mathrm{LiCl}$ either 2 or 12 days before the first test trial. 
as factors. This yielded no significant effects [for the main effect of group, $F<1$; for trials, $F(2,28)=2.83$; and for the group $X$ trials interaction, $F(2,28)=1.99$ ]

These results provide no evidence of recovery of neophobia over a retention interval of 12 days. Apart from the fact that the injection was not paired with saccharin, the animals in this experiment received exactly the same pattern of deprivation, injection, and exposure to the test flavor that was employed in Experiment 1. It is difficult to argue, therefore, that the recovery of the aversion obtained in Experiment 1 could be a consequence of the recovery of neophobia.

\section{EXPERIMENT 3}

The interpretation of the attenuation of latent inhibition after a retention interval offered by interference theory rests on two main assumptions. First, it is assumed that independent memories are established by preexposure and conditioning; second, it is assumed that the memory established by preexposure is sensitive to the effects of the retention interval, whereas that established by conditioning is not. This account can be applied not only to the case in which there is a long retention interval from conditioning to test, but also to the complementary case in which a delay occurs between preexposure and conditioning (with no delay between conditioning and the test). In this latter case, just as in the former, the effects of the preexposure treatment will decline over the retention interval and latent inhibition should again be attenuated. In fact, provided the interval between preexposure and the test is the same, the attenuation produced by the retention interval should be the same in the two cases.

These two cases differ both in the recency of the preexposure experience at the time of conditioning and in the proximity of the conditioning phase to the test, but according to the theory being considered, neither of these factors will be of importance in determining the outcome. The memory formed as a result of the conditioning experience will be the same in both cases, as it is assumed that preexposure is without direct effect on acquisition. The ability of this memory to influence test performance will also be the same in the two cases, given the assumption that the memory of conditioning is impervious to the effects of a retention interval. The critical factor in determining the magnitude of the CR observed in the test session will be the ability of the memory, formed during preexposure, to interfere, and this, in turn, will depend solely on the interval between preexposure and test. Thus, interference theory predicts that latent inhibition should be similarly attenuated by a given retention interval, whether the interval occurs between preexposure and conditioning or between conditioning and testing.

The purpose of Experiment 3, therefore, was to allow us to make a direct comparison of the effects of a long conditioning-to-test interval and a long preexposure-toconditioning interval on latent inhibition. We expected to confirm our previous results for the conditioning-to-test interval, and thus the chief question of interest was whether or not there would be an equivalent attenuation of the latent inhibition effect in the latter case. Previous studies in the taste-aversion paradigm of the effects of interposing an interval between preexposure and conditioning have produced mixed results. Several have shown an attenuation of the latent inhibition effect in these circumstances (e.g., Ackil et al., 1992; Elkins \& Hobbs, 1979; Kraemer \& Roberts, 1984, Experiment 4; McIntosh \& Tarpy, 1977), but there have also been some failures to obtain such an attenuation (e.g., Kalat \& Rozin, 1973; Siegel, 1974), and on one occasion attenuation occurred only when different flavors were used during preexposure and conditioning (Kraemer \& Roberts, 1984, Experiment 4). It is not clear exactly what conditions must be met for an attenuation of latent inhibition to be found in experiments of this sort. But given that the training procedures and parameters used in the present Experiment 1 were effective in producing an attenuation of latent inhibition over the conditioning-to-test interval, it follows from the interference account that they will also be effective when the same interval separates preexposure and conditioning.

\section{Method}

Subjects. The subjects were 24 male Lister rats, with a mean ad-lib weight of $430 \mathrm{~g}$. They were maintained under the same conditions that were used in the previous experiments.

Procedure. The animals were divided into three groups of 8 . All three groups (to be referred to as S-L, L-S, and S-S) were exposed to the saccharin solution for 3 days before conditioning; they had access on each of these days to $10 \mathrm{ml}$ of the solution for a period of $30 \mathrm{~min}$. In Group S-L (short preexposure-conditioning interval, long conditioning-test interval), conditioning took place on the day following the last preexposure day; then there was an interval of 11 days before the first test session. For Group L-S, the conditioning trial occurred 10 days after the last preexposure session; the test session occurred on the day after the postinjection recovery day and thus occurred 2 days after the conditioning trial. These subjects experienced a long preexposure-conditioning interval and a short conditioning-test interval. For both groups, an interval of 12 days elapsed from the last preexposure day to the first test day.

Group S-S experienced conditioning on the day after the last preexposure session and the test on the day following the recovery day (i.e., they received training that was the same as that given to the Pre-2 group of Experiment 1). They served as a control to allow us to assess the extent of the measured aversion when neither of the retention intervals was long. The start of training for Group $S-S$ was delayed so that all three groups came to the test on the same day. As in the previous experiments, all the groups experienced the same schedule of access to fluids so as to ensure that deprivation levels were equated for the test.

Any procedural details not specified here were the same as those described for the previous experiments.

\section{Results and Discussion}

Figure 3 shows mean saccharin consumption over the three test trials for the three groups. Group S-S shows the high level of consumption that would be expected from animals given training known to produce a powerful latent inhibition effect. Both Groups L-S and S-L show an 


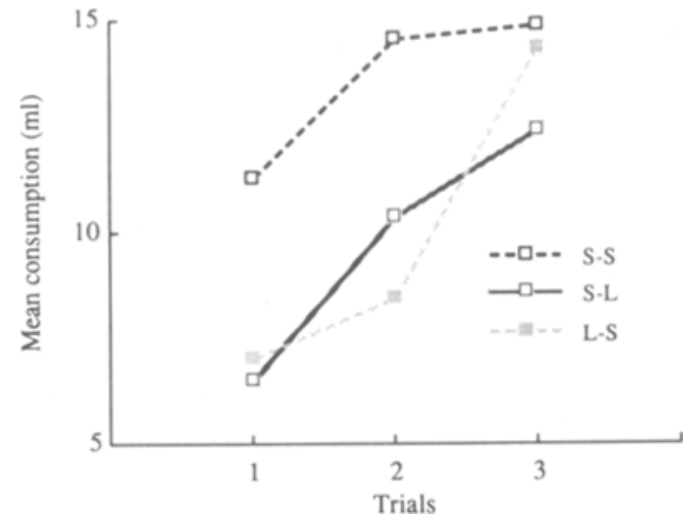

Figure 3. Mean saccharin consumption over the three test trials in Experiment 3. All the subjects received preexposure to saccharin and a conditioning trial. For Groups $S-S$ and $S-L$, the interval between preexposure and conditioning was short, whereas for Group L-S, the interval was long. For Groups S-S and L-S, the interval between conditioning and the test was short, whereas for Group S-L, the interval was long.

apparent attenuation of the latent inhibition effect, and do so to the same extent. An ANOVA performed on the test scores with groups and trials as factors revealed significant main effects of group $[F(2,21)=3.45, p=.05]$ and trial $[F(2,42)=27.99, p<.01]$, and no significant interaction. Pairwise comparisons (Duncan's test) conducted on group means pooled over all three trials showed that Groups L-S and S-L both differed significantly from Group S-S $(p<.05)$, but not from each other.

These results (comparing Groups S-S and S-L) confirm the finding of our previous experiments-that latent inhibition is attenuated when a retention interval intervenes between conditioning and the test. The comparison of Groups S-S and L-S shows that, with these training procedures, the effects of preexposure are also attenuated when a retention interval intervenes between preexposure and conditioning. A comparison of Groups S-L and L-S shows that a 12-day interval between preexposure and testing attenuates latent inhibition to a comparable degree, whether the conditioning episode takes place at the beginning or at the end of that interval. As predicted by the interference account, the recency of the preexposure experience at the time of conditioning plays no role in determining the effects of preexposure. Rather, what counts is the preexposure-test interval, the interval over which the interfering effects of the preexposure experience are presumed to suffer a loss. Finally, the fact that Groups L-S and S-L show a similar degree of aversion in spite of their having experienced different conditioning-test intervals confirms the suggestion that there is no forgetting of the conditioned aversion itself, at least over the interval used in these experiments.

\section{GENERAL DISCUSSION}

In our first experiment, we found that there was a clear attenuation of latent inhibition after a retention interval between conditioning and the test; the animals given conditioning without any prior exposure to the CS showed no change in the strength of the aversion over this interval. These effects were found with a standard training procedure in which the preexposed stimulus was the same as that used as the CS. Experiment 2 allowed us to rule out changes in neophobia as a possible source of the effects seen in Experiment 1. It was shown that neophobia to the flavor used as the CS in Experiment 1 did not recover during a 12-day retention interval in animals receiving the same pattern of exposure to saccharin as the preexposed animals in Experiment 1, but for which saccharin and the $\mathrm{LiCl}$ injection were unpaired. Finally, it was demonstrated in Experiment 3 that an attenuation of latent inhibition comparable to that seen in Experiment 1 can be obtained when the retention interval intervenes between preexposure and conditioning rather than between conditioning and test. The critical determinant of these effects appears to be the length of the interval between preexposure and the test.

Our results are what would be expected on the basis of a retrieval interference theory of latent inhibition. In its most basic form, this theory assumes that the preexposure and conditioning episodes are represented independently in memory and that association occurs normally, even after preexposure. At the time of testing, the memory of preexposure interferes with retrieval of the conditioning episode and the stimulus thus elicits only a weak CR. To accommodate the effects of the retention inter$\mathrm{val}$, it is assumed that the memory of nonreinforced exposure to the stimulus becomes less retrievable with time, so that after a long interval it will no longer be able to interfere with retrieval of the conditioning episode, the effects of which can then be fully expressed.

The retention interval effects demonstrated in our experiments pose a problem, however, for theories that interpret latent inhibition as being the result of an acquisition deficit. Although these theories can accept that an interval between preexposure and conditioning might allow some forgetting of the effects of preexposure, they cannot accommodate the attenuation of the latent inhibition effect produced by an interval between conditioning and the test. There is no reason, according to these theories, why the supposedly weak association formed when conditioning is carried out immediately after preexposure should increase in strength over such a retention interval.

It should be acknowledged that our results do not entirely exclude the possibility that an acquisition deficit plays some role in latent inhibition. It is possible that preexposure might both hinder the acquisition of associative strength and also establish a memory that interferes with the expression of that strength on test. A hybrid theory of this type has certain explanatory advantages (see Hall, 1991); in addition, it supplies a ready explanation for the observation that even at long retention intervals, the latent inhibition effect is not abolished but merely attenuated. But it means the addition of a major new mechanism to theories that have hitherto employed only the very simplest of principles to deal with retrieval. 
Retrieval interference theory is not itself without problems, however. First, there remains some doubt about the generality of the attenuation of latent inhibition after a retention interval. As we have already noted, most demonstrations have found this attenuation only in what may be called generalized latent inhibition, in which different stimuli are used during preexposure and conditioning. Although in our experiments we have been able to obtain the effect in the standard latent inhibition procedure, the reason why this has not been the case in some previous reports (Kraemer \& Ossenkopp, 1986; Kraemer \& Spear, 1992) remains unclear. Further, there is little evidence as yet to demonstrate the generality of these effects across experimental paradigms. Apart from conditioned taste aversion, attenuation of latent inhibition over a retention interval has been shown only with the conditioned emotional response procedure (Kraemer et al., 1991) and the impact of this demonstration is dulled by the presence of differences between the preexposed and nonpreexposed groups in the effect of the different retention intervals on baseline activity. Whether the attenuation of latent inhibition after a retention interval is a general learning phenomenon remains to be determined.

A second concern arises from the undeveloped status of the retrieval theory. It would be helpful, for instance, if we had available a precise specification of the nature of the memory that is held to be formed during preexposure; again, we have no clear statement about what changes take place over a retention interval, about why the memory of conditioning appears to be immune to these effects, and about the exact nature of the interference process itself. These matters need to be settled before a fully satisfactory account of latent inhibition can be offered. Nonetheless, it remains the case that our results are difficult to explain in terms of theories that attribute latent inhibition entirely to an acquisition deficit. And even if it is accepted that there are reasons for retaining certain aspects of these theories (see, e.g., Hall, 1991), it seems likely that it will be necessary to combine them in some way with the proposal that interference at retrieval plays a role in generating the latent inhibition effect.

\section{REFERENCES}

Ackil, J. K., Carman, H. M., Bakner, L., \& Riccio, D. C. (1992). Reinstatement of latent inhibition following a reminder treatment in a conditioned taste aversion paradigm. Behavioral \& Neural Biology, 58, 232-235.

Bakner, L., Strohen, K., Nordeen, M., \& Riccio, D. C. (1991). Postconditioning recovery from the latent inhibition effect in conditioned taste aversion. Physiology \& Behavior, 50, 1269-1272.

BAtsell, W. R., \& Best, M. R. (1992). Variations in the retention of taste aversions: Evidence for retrieval competition. Animal Learming \& Behavior, 20, 146-159.

Bouton, M. E. (1991). Context and retrieval in extinction and in other examples of interference in simple associative learning. In L. W. Dachowski \& C. F. Flaherty (Eds.), Current topics in animal learning: Brain, emotion and cognition (pp. 25-53). Hillsdale, NJ: Erlbaum.
BROOKSHIRE, K. H., \& BRACKBILL, R. M. (1976). Formation and retention of conditioned taste aversions and UCS habituation. Bulletin of the Psychonomic Society, 7, 125-128.

Colby, J. J., SMITH, N. F. (1977). The effect of three procedures for eliminating a conditioned taste aversion in the rat. Learning \& Motivation, 8, 404-413.

Domjan, M. (1977). Attenuation and enhancement of neophobia for edible substances. In L. M. Barker, M. R. Best, \& M. Domjan (Eds.), Learning mechanisms in food selection (pp. 151-180). Waco, TX: Baylor University Press.

ElKINS, R. L., \& HoBBs, S. H. (1979). Forgetting, preconditioning CS familiarization and taste aversion learning: An animal experiment with implications for alcoholism treatment. Behavioral Research \& Therapy, 17, 567-573.

HaLL, G. (1991). Perceptual and associative learning. Oxford: Clarendon.

Kalat, J. W., \& Rozin, P. (1973). "Learned safety" as a mechanism in long-delay taste aversion in rats. Journal of Comparative \& Physiological Psychology, 83, 198-207.

Kasprow, W. J., Catterson, D., Schachtman, T. R., \& Miller, R. R. (1984). Attenuation of latent inhibition by postacquisition reminder. Quarterly Journal of Experimental Psychology, 36B, 53-63.

Kraemer, P. J., Hoffmann, H., \& Spear, N. E. (1988). Attenuation of the CS-preexposure effect after a retention interval in preweanling rats. Animal Learning \& Behavior, 16, 185-190.

KraEmer, P. J., \& OSSENKOPP, K.-P. (1986). The effects of flavor preexposure and test interval on conditioned taste aversions in rats. Bulletin of the Psychonomic Society, 24, 219-221.

Kraemer, P. J., Randall, C. K., Carbary, T. J. (1991). Release from latent inhibition with delayed testing. Animal Learning \& Behavior, 19, 139-145.

Kraemer, P. J., \&oberts, W. A. (1984). The influence of flavor preexposure and test interval on conditioned taste aversions in the rat. Learning \& Motivation, 15, 259-278.

Kraemer, P. J., Spear, N. E. (1992). The effect of nonreinforced stimulus exposure on the strength of a conditioned taste aversion as a function of retention interval: Do latent inhibition and extinction involve a shared process? Animal Learning \& Behavior, 20, 1-7.

LuBow, R. E. (1973). Latent inhibition. Psychological Bulletin, 79, 398-407.

LuBow, R. E. (1989). Latent inhibition and conditioned attention theory. Cambridge: Cambridge University Press.

Lubow, R. E., WeINER, I., \& SchnUR, P. (1981). Conditioned attention theory. In G. H. Bower (Ed.), The psychology of learning and motivation (Vol. 15, pp. 1-49). New York: Academic Press.

MACKINTOSH, N. J. (1975). A theory of attention: Variations in the associability of stimuli with reinforcement. Psychological Review, 82, 276-298.

McINTOSH, S. M., \& TARPY, R. M. (1977). Retention of latent inhibition in a taste-aversion paradigm. Bulletin of the Psychonomic Society, 9, 411-412.

Pearce, J. M., \& Hall, G. (1980). A model for Pavlovian learning: Variations in the effectiveness of conditioned but not of unconditioned stimuli. Psychological Review, 87, 532-552.

SIEGEL, S. (1974). Flavor preexposure and "learned safety." Journal of Comparative \& Physiological Psychology, 87, 1073-1082.

THOMPSON, R. F., \& SPENCER, W. A. (1966). Habituation: A model phenomenon for the study of neuronal substrates of behavior. Psychological Review, 73, 16-43.

WAGNER, A. R. (1976). Priming in STM: An information processing mechanism for self-generated or retrieval-generated depression in performance. In T. J. Tighe \& R. N. Leaton (Eds.), Habituation: Perspectives from child development, animal behavior, and neurophysiology (pp. 95-128). Hillsdale, NJ: Erlbaum.

(Manuscript received June 28, 1993; revision accepted for publication September 14, 1993.) 